\title{
Independent risk factors for postoperative AKI and the impact of the AKI on 30-day postoperative outcomes in patients with type A acute aortic dissection: an updated meta-analysis and meta- regression
}

\author{
Jiayang Wang ${ }^{1,2 \#}$, Wenyuan Yu ${ }^{1}$, Guangyao Zhai ${ }^{3 \#}$, Nan Liu ${ }^{2}$, Lizhong Sun ${ }^{1}$, Junming Zhu ${ }^{1}$ \\ ${ }^{1}$ Department of Cardiac Surgery, ${ }^{2}$ Center for Cardiac Intensive Care, ${ }^{3}$ Department of Cardiology, Beijing An Zhen Hospital Capital Medical \\ University, Beijing 100029, China \\ Contributions: (I) Conception and design: J Wang, N Liu; (II) Administrative support: J Zhu; (III) Provision of study materials or patients: G Zhai; (IV) \\ Collection and assembly of data: W Yu, J Zhu; (V) Data analysis and interpretation: L Sun; (VI) Manuscript writing: All authors; (VII) Final approval \\ of manuscript: All authors. \\ \#These authors contributed equally to this work. \\ Correspondence to: Nan Liu, MD. Center for Cardiac Intensive Care, Beijing An Zhen Hospital Capital Medical University, Beijing 100029, China. \\ Email: ln9102@126.com; Junming Zhu, MD. Department of Cardiac Surgery, Beijing An Zhen Hospital Capital Medical University, Beijing 100029, \\ China. Email: anzhenzjm@163.com.
}

Background: This meta-analysis aims to investigate the effects of postoperative acute kidney injury (AKI) on 30-day postoperative outcomes and the independent risk factors for postoperative AKI in patients with type A acute aortic dissection (TAAD).

Methods: Relevant reports published between January 1, 2011 and May 31, 2017 were searched in multiple electronic literature databases. A total of seven eligible articles were included in the meta-analysis.

Results: Postoperative AKI was associated with $249 \%$ increase in 30-day postoperative mortality [odds ratio (OR): 3.49; 95\% confidence interval (CI): 2.17-5.59; $\mathrm{P}<0.0001]$. Subgroup analysis revealed that patients with stage II/III AKI showed $445 \%$ increase in 30-day postoperative mortality compared with the control group (OR: 5.45; 95\% CI: 2.87-10.36; P<0.0001). Postoperative AKI was also associated with $143 \%, 432 \%$, and $126 \%$ increase in the incidences of 30-day postoperative stroke, bleeding, and respiratory complications, respectively. Notably, high body mass index (BMI), advanced age, and perioperative sepsis were independent risk factors for postoperative AKI in patients with TAAD.

Conclusions: This meta-analysis firstly provided clinical evidence showing the adverse effects of postoperative AKI on 30-day postoperative outcomes in patients with TAAD and identified high BMI, advanced age, and perioperative sepsis as the independent risk factors for postoperative AKI. These findings suggest that preventive or therapeutic methods to effectively manage postoperative AKI may improve 30-day postoperative outcomes in patients with TAAD.

Keywords: Acute kidney injury (AKI); postoperative; risk factors; 30-day mortality; type A acute aortic dissection (TAAD)

Submitted Sep 19, 2017. Accepted for publication Apr 19, 2018.

doi: $10.21037 /$ jtd.2018.05.47

View this article at: http://dx.doi.org/10.21037/jtd.2018.05.47 


\section{Introduction}

Despite the improvement in medical management and surgical technique, the incidence of 30-day postoperative morbidity and mortality in patients with type A acute aortic dissection (TAAD) remains high (1). Hagan and colleagues reported that the 30 -day postoperative mortality was $27.4 \%$ after surgical repair of TAAD (1). Previous studies have found multiple predictors for adverse postoperative outcomes from surgical repair of TAAD, such as ST-T abnormalities (2,3). However, previous reports investigating the association between postoperative acute kidney injury (AKI) and 30-day postoperative adverse outcomes in patients with TAAD show inconsistent results $(4,5)$. Some studies have shown that only severe postoperative AKI increased 30-day postoperative mortality, whereas others have found that postoperative AKI regardless of the degree of severity worsened the mortality of patients with TAAD (5-8). As far as now, no meta-analysis systematically investigates the effects of postoperative AKI on 30-day mortality. In addition, investigations that focus on the impact of postoperative AKI on the incidences of particular 30-day postoperative morbidities, including adverse cardiovascular events and complications in other organs, are still insufficient (9). Furthermore, the clinical and procedural risk factors for postoperative AKI in patients with TAAD are still unknown. Meta-analyses of the eligible studies demonstrating multivariate logistic regression analysis to identify independent pre- or intraoperative risk factors for postoperative AKI are still lacking. This current study aims to fill the knowledge gap.

Here, we conducted a meta-analysis to investigate the effects of post-AKI on 30-day postoperative mortality and morbidities and examined the independent correlation between pre- or intraoperative variables and postoperative AKI in patients with TAAD. We also performed a metaregression to examine the effects of covariates, which could be potential modifiers, on the end results. Our study firstly provides clinical evidence to support the association of postoperative AKI and poor 30-day postoperative outcomes in patients with TAAD.

\section{Methods}

This meta-analysis was conducted in accordance with the Preferred Reporting Items for Systematic Reviews and Meta-analyses (PRISMA) guidelines (10). Two investigators (J Wang and $\mathrm{W} \mathrm{Yu}$ ) searched the literature databases
PubMed, Ovid Medline, and EMBASE independently to identify reports on the independent risk factors for postoperative AKI in patients with TAAD and the impact of the AKI on 30-day postoperative outcomes. Studies with the following characteristics were excluded: (I) in the format of an abstracts, editorial, letter, review, comment, or case report; (II) with a sample size <30; (III) using duplicate data; (IV) not on human subjects. The following search terms were used: ("acute type A aortic dissection" OR "TAAD”) AND ("risk factors" OR "predictors" OR "30-day postoperative mortality" OR "30-day postoperative stroke" OR "30-day postoperative complications") AND ("acute kidney injury" OR "AKI” OR "acute renal failure" OR "acute renal complication"). Most of the relevant articles were published after 2011. The majority of the relevant reports published before 2011 are review articles but not original clinical studies. In addition, surgical technique and procedures have evolved rapidly. Including articles published before 2011 may increase study heterogeneity in the meta-analysis and thus compromise the quality of the meta-analysis. Therefore, to reduce study heterogeneity, the date of publication was restricted between January 1 , 2011 and May 31, 2017.

Downs and Black score was used to estimate the quality of each included study, and the quality of a report with a score $\geq 20$ was considered satisfactory and $<20$ was considered poor. The primary endpoints were the incidence rates of 30-day postoperative mortality and 30-day postoperative stroke and bleeding. Odds ratio (OR) with $95 \%$ confidence interval $(95 \% \mathrm{CI})$ was calculated. The study heterogeneity was assessed by the $\mathrm{I}^{2}$ index, and $\mathrm{I}^{2}>50 \%$ represents a statistically significant heterogeneity. Publication bias was estimated by Egger's weighted regression. Meta-regression was conducted to evaluate the effects of potentially meaningful predictors of AKI on the meta-analysis. A Q model statistic was used in the metaregression. All the analyses were performed using the STATA statistical analysis software. $P$ value was 2 -sided and $\mathrm{P}<0.05$ was considered statistically significant.

\section{Results}

The study selection process is displayed in Figure 1. A total of seven articles met the inclusion criteria (5-8,11-13), and 1,621 patients were included in the current meta-analysis. The risk of bias assessment is presented in Table 1. Downs and Black score analysis revealed that the score was between 20 and 25 ; all articles had good quality (score $\geq 20$ ). 


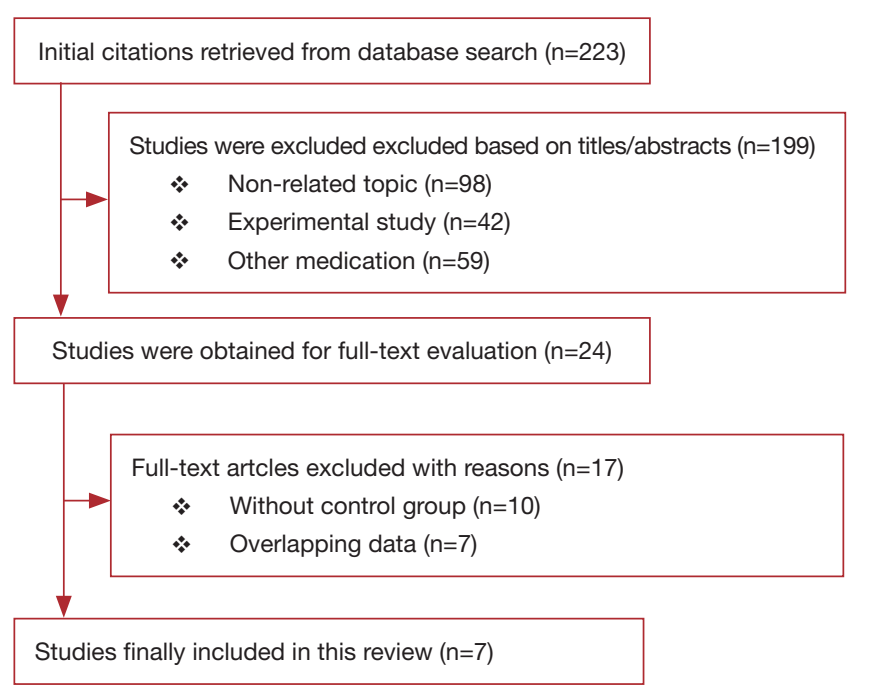

Figure 1 Flow diagram of study selection.
Patients with postoperative AKI showed 249\% increase in 30-day postoperative mortality compared with the control group (OR: 3.49; 95\% CI: 2.17-5.59; $\mathrm{P}<0.0001$; Figure 2). No significant study heterogeneity $\left(\mathrm{I}^{2}=0.0 \%\right.$; $\mathrm{P}=0.492)$ and publication bias $(\mathrm{P}=0.156)$ were found in the articles included in the analysis. The meta-regression revealed that the meta-analysis result was not affected by the different AKI definitions used in the reports and the publication year of the included reports (Figure $3 A, B$ ). Subgroup analysis found that patients with stage II/III AKI demonstrated $445 \%$ increase in 30-day postoperative mortality compared with the control group (OR: $5.45 ; 95 \%$ CI: 2.87-10.36; $\mathrm{P}<0.0001$; Figure 2B). Compared with the control group, patients with postoperative AKI had $143 \%$ increase in the incidence of 30-day postoperative stroke (OR: 2.43; 95\% CI: 1.52-3.87; $\mathrm{P}<0.0001$; Figure 4A), 432\%

Table 1 Studies included in the systematic review

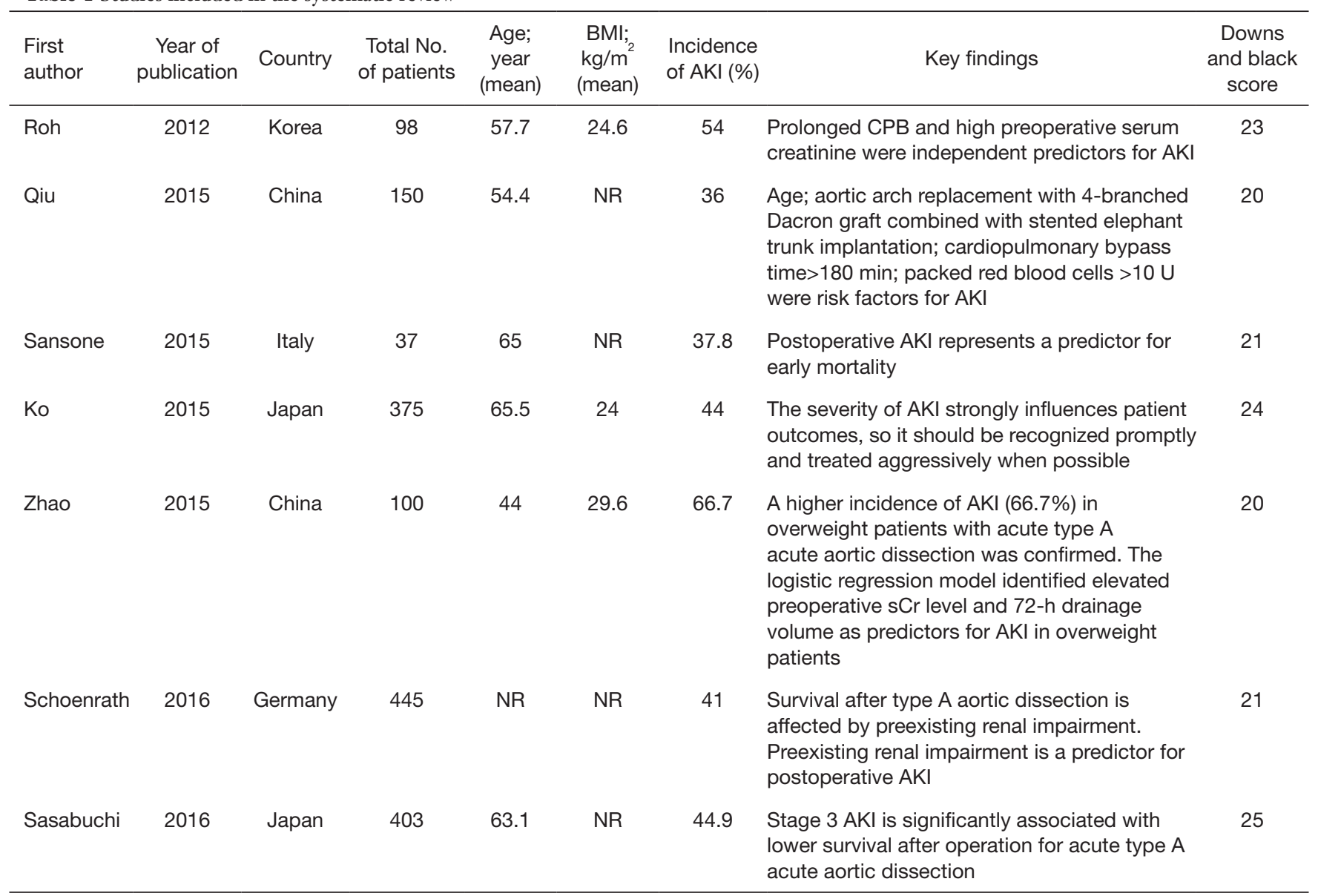

AKI, acute kidney injury; CPB, cardiopulmonary bypass; NR, not report. 

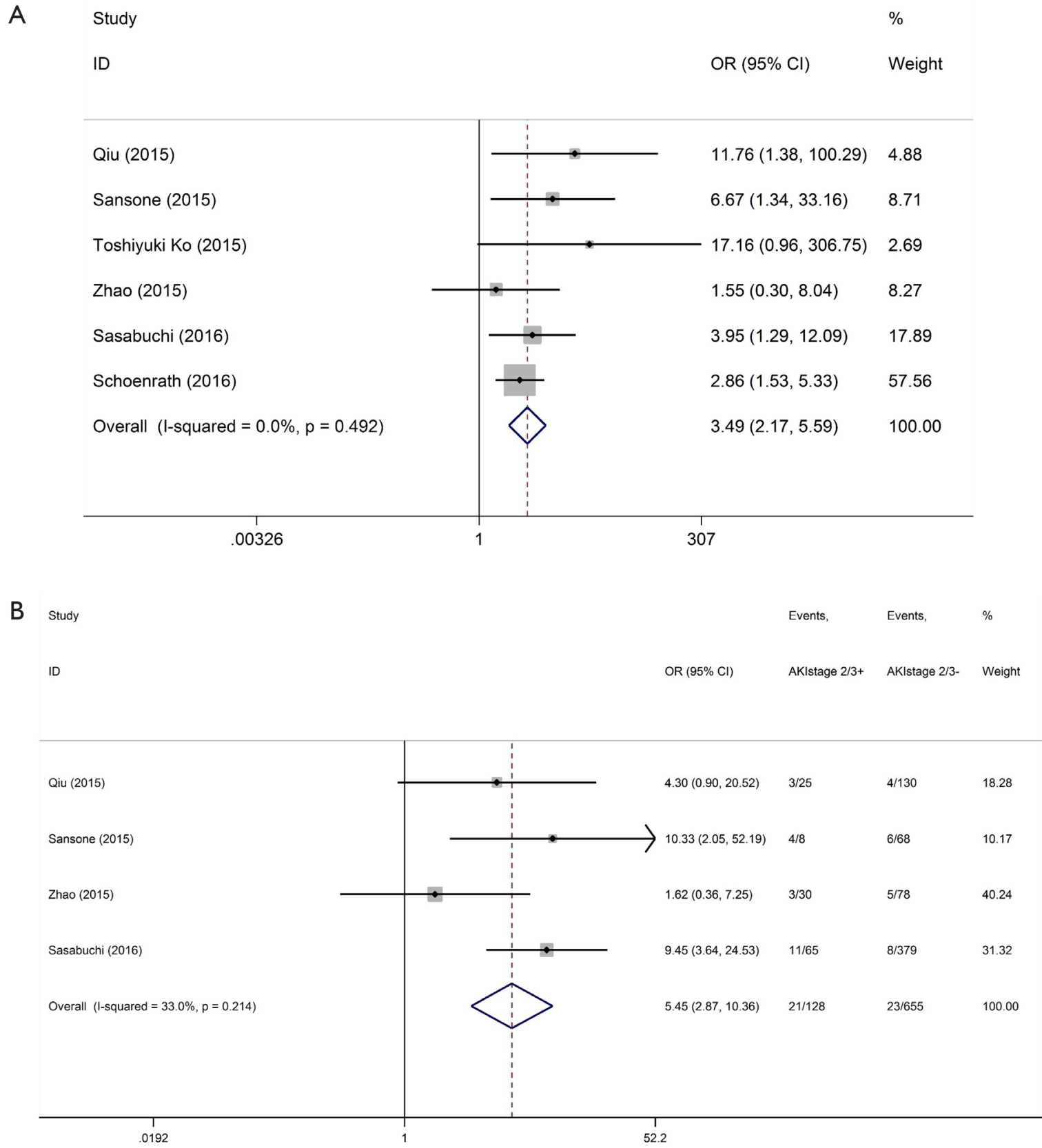

Figure 2 Forest plots showing that postoperative AKI increased 30-day postoperative mortality. (A) Patients with postoperative AKI showed $249 \%$ increase in 30-day postoperative mortality compared with the control group; (B) the AKI stage II/III subgroup showed $445 \%$ increase in 30-day postoperative mortality compared with the control group. AKI, acute kidney injury.

increase in the incidence of 30-day postoperative bleeding (OR: 5.32; 95\% CI: 2.11-13.42; $\mathrm{P}<0.0001$; Figure $4 B$ ), and $126 \%$ increase in the incidence of 30 -day postoperative respiratory complication (OR: 2.26; 95\% CI: 1.39-3.65; $\mathrm{P}=0.001$; Figure 4 ).
We further investigated the independent risk factors for postoperative AKI in patients with TAAD. High body mass index (BMI) (OR: 1.26; 95\% CI: 1.02-1.55; $\mathrm{P}=0.031$ ), advanced age (OR: 1.26; 95\% CI: 1.11-1.43; $\mathrm{P}<0.0001$ ), and perioperative sepsis [defined by 2001 SCCM/ 

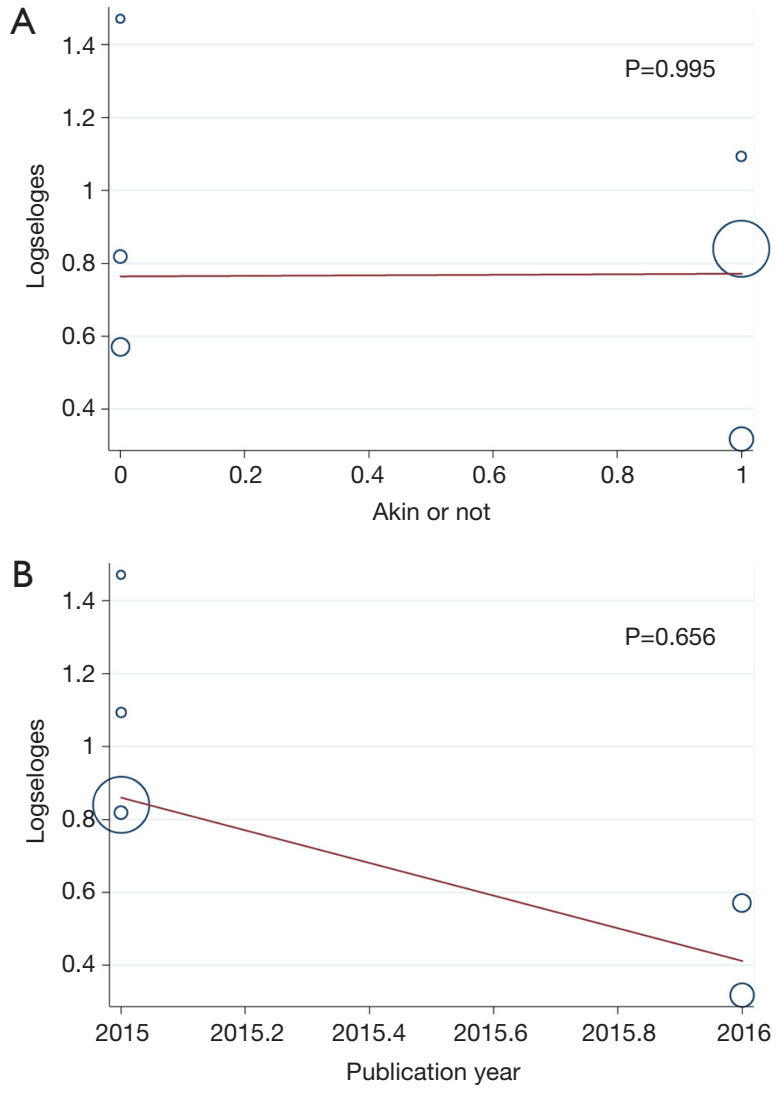

Figure 3 Meta-regression revealed that postoperative AKIassociated increase in 30-day postoperative mortality was not influenced by different AKI definition used in the included studies (A) and publication year of the included studies (B). AKI, acute kidney injury.

ESICM/ACCP/ATS/SIS International Sepsis Definitions Conference (14)] (OR: 1.92; 95\% CI: 1.13-3.29; P=0.017) were independently associated with postoperative AKI (Figure 5). The included studies showed no significant heterogeneity.

\section{Discussion}

To the best of our knowledge, this is the first metaanalysis to investigate the effects of postoperative AKI on 30-day postoperative outcomes and examine the independent risk factors for postoperative AKI in patients undergoing surgical repair for TAAD. Our key findings are: (I) postoperative AKI, in particular stage II/III AKI, was associated with a significant increase in 30-day postoperative mortality; (II) postoperative AKI was also associated with a significant increase in the incidences of 30-day postoperative stroke, bleeding events, and respiratory complications; (III) high BMI, advanced age, and perioperative sepsis were independent risk factors for postoperative AKI.

Postoperative AKI is a well-known serious complication in patients undergoing surgical repair of TAAD, and the reported incidence of the condition is higher than $20 \%(8,15,16)$. Zhao and colleagues have suggested that postoperative AKI might increase the risk of 10 -year mortality regardless of renal recovery at discharge but might not significantly influence 30-day mortality after surgical repair of TAAD (6). Ko and colleagues have found a positive correlation between the degree of AKI severity and the incidence rate of 30-day mortality (11), and they have shown that stage III AKI was associated with a significant increase in the 30-day mortality in their patient cohort but stages I and II AKI were not (11). In contrast to the report by Toshiyuki and colleagues, others have indicated that postoperative AKI regardless of the degree of severity might increase the 30-day mortality after surgical repair of TAAD $(7,8,12)$. Furthermore, Sasabuchi and colleagues have found that postoperative AKI increased the incidence of 30-day postoperative bleeding but had no effects on the incidence of 30-day postoperative stroke (5). Our results support that postoperative AKI, in particular stage II and III AKI, increases the incidences of 30-day postoperative mortality, stroke, bleeding events, and respiratory complications in patients undergoing surgical repair of TAAD.

The mechanism underlying the postoperative AKIassociated 30-day mortality after surgical repair of TAAD may be related to postoperative AKI-induced chronic kidney disease and end-stage renal dysfunction. A previous metaanalysis has shown that patients with AKI had higher risk of developing chronic kidney disease and end-stage renal dysfunction (17). Olsson and colleagues have demonstrated that organ functional failure was strongly related to inhospital mortality in patients undergoing surgical repair of TAAD (18). Additionally, Fann and colleagues have found that renal dysfunction per se was a significant independent predictor of operative death (19). Indeed, previous studies have shown that the operative mortality rate in patients with renal ischemia was $50 \%$ whereas the mortality was $23 \%$ for those whose renal perfusion was not compromised $(19,20)$. Similarly, Estrera and colleagues have believed that insufficient perfusion in the end organs such as kidney might be the most common cause for significant morbidities during the acute presentation of aortic dissection (21). 

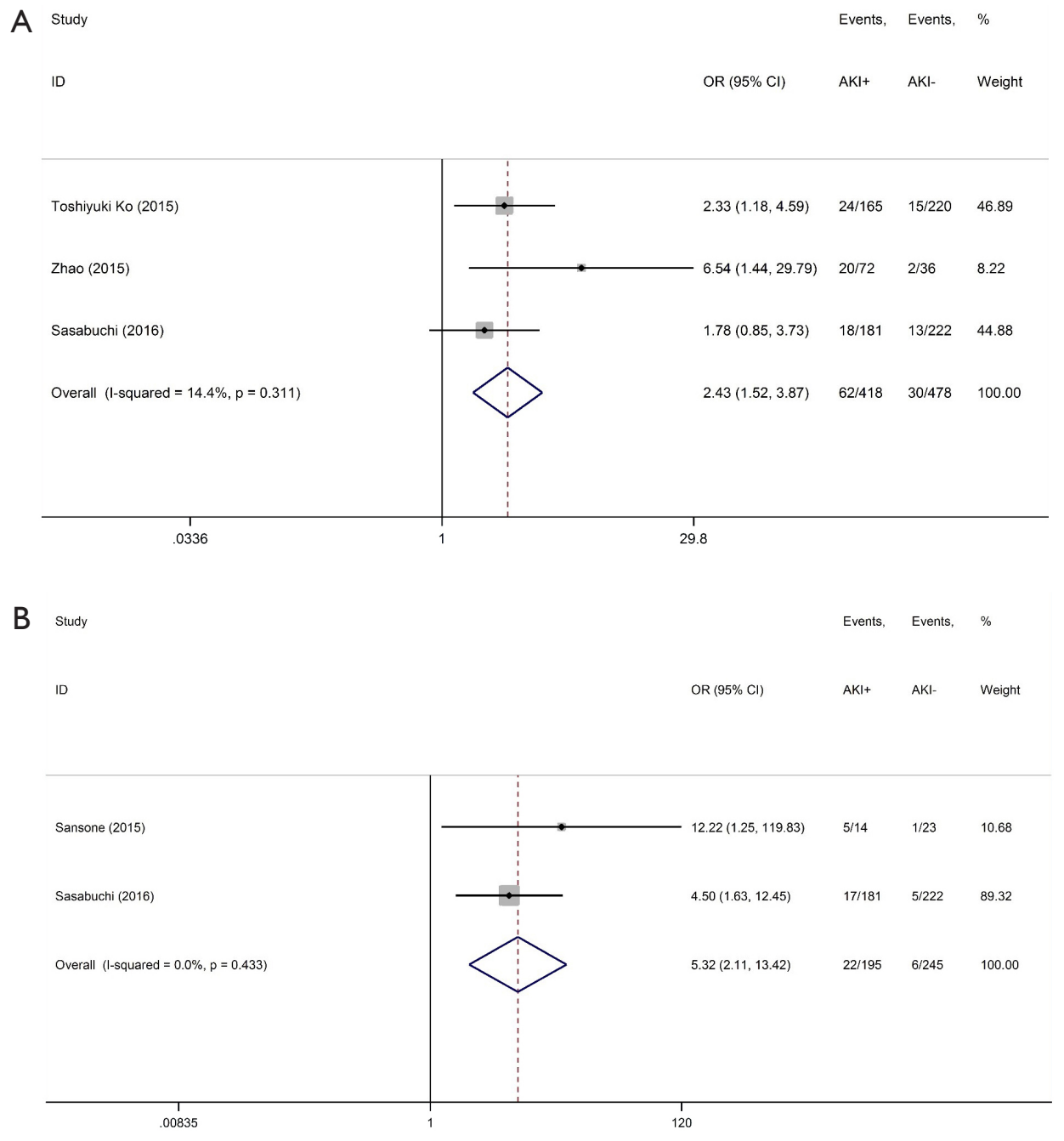

C Study

$\%$

ID

OR $(95 \% \mathrm{Cl}) \quad$ Weight

Qiu (2015)

Sansone (2015)

Toshiyuki Ko (2015)

Overall $(I-$ squared $=38.9 \%, p=0.195$ )

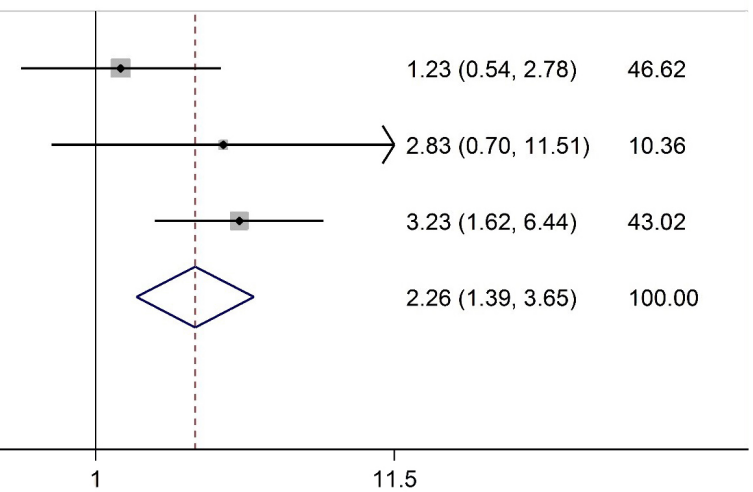

Figure 4 Forest plots showing that compared with the control group, postoperative AKI was associated with $143 \%$, $432 \%$, and $126 \%$ increase in the incidences of 30-day postoperative stroke (A), bleeding (B), and respiratory complication (C), respectively. 


\begin{tabular}{|c|c|c|c|c|c|}
\hline Varlables & & OR(95\% CI) & $\mathbf{Z}$ & P-Valve & $I^{2}(\%)$ \\
\hline Intraoperative RBC transfusion & $10-1$ & $0.98(0.92,1.05)$ & 0.58 & 0.559 & 10.5 \\
\hline Hypertension & $\longrightarrow$ & $1.20(0.71,2.03)$ & 0.68 & 0.499 & 0.00 \\
\hline Preoperative sCr Level & tor & $1.03(0.98,1.08)$ & 1.19 & 0.233 & 72.8 \\
\hline Perioperative Sepsis & $\longmapsto$ & $1.92(1.13,3.29)$ & 2.39 & 0.017 & 15.2 \\
\hline BMI & $\longmapsto$ & $1.26(1.02,1.55)$ & 2.16 & 0.031 & 42.7 \\
\hline Advancing Age & $\longmapsto$ & $1.26(1.11,1.43)$ & 3.55 & 0.000 & 38.4 \\
\hline
\end{tabular}

Figure 5 BMI, advanced age, and perioperative sepsis were independent risk factors for postoperative AKI. AKI, acute kidney injury; BMI, body mass index.

Thus, patients with postoperative AKI may likely develop chronic kidney disease or end-stage renal dysfunction, which subsequently increase the incidences of 30-day postoperative mortality and morbidities.

Identification of the independent risk factors for postoperative AKI in patients undergoing surgical repair of TAAD is essential to prevent the AKI and improve postoperative outcomes. In the current meta-analysis, perioperative sepsis was found to be an independent risk factor for postoperative AKI after surgical repair of TAAD. Consistent with our finding, in a multinational, prospective, observational study including 29,269 critically ill patients, Uchino and colleagues reported that sepsis contributed to AKI development substantially (22). Sepsis may cause renal microvasculature dysfunction, such as intra-renal hemodynamic changes, endothelial dysfunction, and intraglomerular thrombosis, leading to postoperative AKI after surgical repair of TAAD (22-24). In addition, sepsis induces strong inflammatory responses, which in turn promote significant cytokine secretion $(25,26)$. Thus, sepsis causes cytokine storm and hemodynamic instability, ultimately resulting in AKI (24). In addition to the supportive treatments including fluid management, antibiotics, vasopressors, diuretics, and dialysis (27), anti-TNF therapy has recently been tested to treat sepsis-induced postoperative AKI in animal models and shown promising results $(28,29)$. Although showing effectiveness in animal models, many anti-inflammatory drugs fail in clinical trials. Effective therapies to manage sepsis-induced postoperative AKI are still unavailable.

The current study also found that high BMI and advanced age were independent risk factors for postoperative AKI. Contrarily, intraoperative RBC transfusion, hypertension, preoperative serum Cr level did not correlate with postoperative AKI in patients undergoing surgical repair of
TAAD. Previous studies on the risk factors for postoperative AKI have shown various results. In a retrospective study including 108 consecutive overweight patients (BMI $\geq 24$ ) with TAAD, Zhao and colleagues have found no correlation between advanced age and postoperative AKI (6). Roh and colleagues have also reported that advanced age and $\mathrm{BMI}$ were not independent risk factors for postoperative AKI (8). However, Ko and colleagues have shown that high $\mathrm{BMI}$ was an independent risk factor for postoperative AKI in patients with TAAD (11). Obesity-induced hemodynamic perturbations may explain the association between high BMI and the development of postoperative AKI (30). Obese patients often have Cor pulmonale condition because of hypoventilation, sleep apnea, and pulmonary hypertension $(31,32)$, leading to sodium avidity and peripheral venous congestion. Peripheral venous congestion can increase renal venous pressure and thus decrease urine formation (33). A recent clinical study has found that high central venous pressure is associated with renal dysfunction. In fact, increasing central venous pressure has been found to be associated with sepsis-induced postoperative AKI in patients undergoing surgical repair of TAAD (33).

Aging could deteriorate renal function by causing adverse structural and functional changes in the kidney, such as parenchymal mass loss, progressive glomerulosclerosis, tubulopathy, interstitial fibrosis, and afferent-efferent arteriolar shunts (34). Thus, agingmediated adverse renal structural and functional changes may contribute to the development of postoperative AKI after surgical repair of TAAD.

In addition to therapies to manage sepsis-induced postoperative AKI, effective management of medullary ischemia may also improve patient outcomes. Renal damages can cause medullary ischemia or reperfusion injury (hypothermic circulatory arrest -mediated). Medullary 
blood flow reduction accompanied with hypoxia may lead to a failure of osmotic gradient regulation (13). Shorter hypothermic circulatory arrest and lesser hypothermia may result in better renal outcome, especially for elderly patients (13).

The limitations of the current study are: (I) because of the limited information on the surgical procedure to treat TAAD in the included reports, we were unable to analyze the impact of surgical procedure on the incidence of postoperative AKI; (II) subgroup analysis based on different AKI definition is lacking due to the small number of eligible reports included in the current meta-analysis although our meta-regression found no significant effects of AKI definition on the meta-analysis results; (III) all of the included studies were observational studies. Highquality randomized controlled trials are required to further confirm the findings; (IV) all of the included articles did not specify type I or type II ascending aortic dissection in the patient inclusion criteria. Thus, we were not able to further compare the effects of type I versus type II ascending aortic dissections on renal injury. (V) We could not determine whether both pulmonary issues and postoperative AKI could be associated with higher mortality and/or morbidity than just AKI alone in the current study. According to our literature search, we have not found any study investigating mortality and/or morbidity of patients with both pulmonary issues and postop AKI.

\section{Conclusions}

This meta-analysis firstly revealed that postoperative AKI, in particular severe AKI, was associated with significantly increased incidences of 30-day postoperative mortality, stroke, bleeding events, and respiratory complications in patients undergoing surgical repair of TAAD. High BMI, advanced age, and perioperative sepsis were found to be independently correlated with the postoperative AKI. Thus, effective management of obesity and perioperative sepsis may prevent postoperative AKI and thus improve 30-day postoperative outcomes from surgical repair of TAAD.

\section{Acknowledgements}

Funding: This study was supported by the Foundation of Beijing Anzhen Hospital, Capital Medical University (No. 2016Z01) and National Science and Technology Support Program of China (No. 2015BAI12B03).

\section{Footnote}

Conflicts of Interest: The authors have no conflicts of interest to declare.

\section{References}

1. Hagan PG, Nienaber CA, Isselbacher EM, et al. The International Registry of Acute Aortic Dissection (IRAD): new insights into an old disease. JAMA 2000;283:897-903.

2. Hoff E, Eagle T, Pyeritz RE, et al. Pulse pressure and type A acute aortic dissection in-hospital outcomes (from the International Registry of Acute Aortic Dissection). Am J Cardiol 2014;113:1255-9.

3. Kosuge M, Uchida K, Imoto K, et al. Frequency and implication of ST-T abnormalities on hospital admission electrocardiograms in patients with type A acute aortic dissection. Am J Cardiol 2013;112:424-9.

4. Nienaber CA, Eagle KA. Aortic dissection: new frontiers in diagnosis and management: Part II: therapeutic management and follow-up. Circulation 2003;108:772-8.

5. Sasabuchi Y, Kimura N, Shiotsuka J, et al. Long-Term Survival in Patients With Acute Kidney Injury After Acute Type A Aortic Dissection Repair. Ann Thorac Surg 2016;102:2003-9.

6. Zhao H, Pan X, Gong Z, et al. Risk factors for acute kidney injury in overweight patients with acute type A aortic dissection: a retrospective study. J Thorac Dis 2015;7:1385-90.

7. Schoenrath F, Laber R, Maralushaj M, et al. Survival, Neurologic Injury, and Kidney Function after Surgery for Acute Type A Aortic Dissection. Thorac Cardiovasc Surg 2016;64:100-7.

8. Roh GU, Lee JW, Nam SB, et al. Incidence and risk factors of acute kidney injury after thoracic aortic surgery for acute dissection. Ann Thorac Surg 2012;94:766-71.

9. Ryden L, Ahnve S, Bell M, et al. Acute kidney injury after coronary artery bypass grafting and long-term risk of myocardial infarction and death. Int J Cardiol 2014;172:190-5.

10. Moher D, Liberati A, Tetzlaff J, et al. Preferred reporting items for systematic reviews and metaanalyses: the PRISMA statement. Ann Intern Med 2009;151:264-9, W64.

11. Ko T, Higashitani M, Sato A, et al. Impact of Acute Kidney Injury on Early to Long-Term Outcomes in Patients Who Underwent Surgery for Type A Acute Aortic Dissection. Am J Cardiol 2015;116:463-8. 
12. Qiu Z, Chen L, Cao H, et al. Analysis of risk factors for acute kidney injury after ascending aortic replacement combined with open placement of triple-branched stent graft in type A aortic dissection: a new technique versus the traditional method in a single Chinese center. Med Sci Monit 2015;21:674-80.

13. Sansone F, Morgante A, Ceresa F, et al. Prognostic Implications of Acute Renal Failure after Surgery for Type A Acute Aortic Dissection. Aorta (Stamford) 2015;3:91-7.

14. Levy MM, Fink MP, Marshall JC, et al. 2001 SCCM/ ESICM/ACCP/ATS/SIS International Sepsis Definitions Conference. Crit Care Med 2003;31:1250-6.

15. Sinatra R, Melina G, Pulitani I, et al. Emergency operation for acute type A aortic dissection: neurologic complications and early mortality. Ann Thorac Surg 2001;71:33-8.

16. Collins JS, Evangelista A, Nienaber CA, et al. Differences in clinical presentation, management, and outcomes of acute type a aortic dissection in patients with and without previous cardiac surgery. Circulation 2004;110:II237-42.

17. Coca SG, Singanamala S, Parikh CR. Chronic kidney disease after acute kidney injury: a systematic review and meta-analysis. Kidney Int 2012;81:442-8.

18. Olsson C, Franco-Cereceda A. Impact of organ failure and major complications on outcome in acute Type A aortic dissection. Scand Cardiovasc J 2013;47:352-8.

19. Fann JI, Smith JA, Miller DC, et al. Surgical management of aortic dissection during a 30-year period. Circulation 1995;92:II113-21.

20. Fann JI, Sarris GE, Mitchell RS, et al. Treatment of patients with aortic dissection presenting with peripheral vascular complications. Ann Surg 1990;212:705-13.

21. Estrera AL, Miller CC, Goodrick J, et al. Update on outcomes of acute type B aortic dissection. Ann Thorac Surg 2007;83:S842-5; discussion S6-50.

22. Uchino S, Kellum JA, Bellomo R, et al. Acute renal failure in critically ill patients: a multinational, multicenter study.
JAMA 2005;294:813-8.

23. Wan L, Bagshaw SM, Langenberg C, et al. Pathophysiology of septic acute kidney injury: what do we really know? Crit Care Med 2008;36:S198-203.

24. Zarjou A, Agarwal A. Sepsis and acute kidney injury. J Am Soc Nephrol 2011;22:999-1006.

25. Hotchkiss RS, Karl IE. The pathophysiology and treatment of sepsis. N Engl J Med 2003;348:138-50.

26. Munford RS, Pugin J. Normal responses to injury prevent systemic inflammation and can be immunosuppressive. Am J Respir Crit Care Med 2001;163:316-21.

27. De Vriese AS, Bourgeois M. Pharmacologic treatment of acute renal failure in sepsis. Curr Opin Crit Care 2003;9:474-80.

28. Reinhart K, Karzai W. Anti-tumor necrosis factor therapy in sepsis: update on clinical trials and lessons learned. Crit Care Med 2001;29:S121-5.

29. Cunningham PN, Dyanov HM, Park P, et al. Acute renal failure in endotoxemia is caused by TNF acting directly on TNF receptor-1 in kidney. J Immunol 2002;168:5817-23.

30. Chagnac A, Weinstein T, Korzets A, et al. Glomerular hemodynamics in severe obesity. Am J Physiol Renal Physiol 2000;278:F817-22.

31. Marik PE, Desai H. The emergent malignant obesity hypoventilation syndrome: a new critical care syndrome. Author reply. J Intensive Care Med 2013;28:200.

32. Marik PE, Desai H. Characteristics of patients with the "malignant obesity hypoventilation syndrome" admitted to an ICU. J Intensive Care Med 2013;28:124-30.

33. Danziger J, Chen KP, Lee J, et al. Obesity, Acute Kidney Injury, and Mortality in Critical Illness. Critical Care Medicine 2016;44:328-34.

34. Cowen LE, Hodak SP, Verbalis JG. Age-associated abnormalities of water homeostasis. Endocrinol Metab Clin North Am 2013;42:349-70.
Cite this article as: Wang J, Yu W, Zhai G, Liu N, Sun L, Zhu J. Independent risk factors for postoperative AKI and the impact of the AKI on 30-day postoperative outcomes in patients with type A acute aortic dissection: an updated meta-analysis and meta-regression. J Thorac Dis 2018;10(5):2590-2598. doi: $10.21037 /$ jtd.2018.05.47 